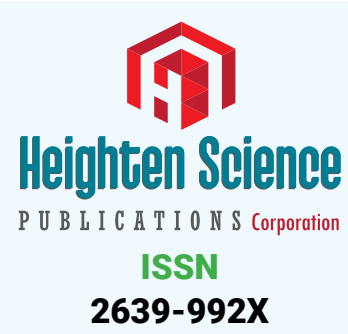

*Address for Correspondence: Sulaiman M Al-Mayouf, MD, Consultant and Section Head, Rheumatology, Department of Pediatrics, King Faisal Specialist Hospital and Research Center, Alfaisal University, Po Box 3354, Riyadh 11211, Saudi Arabia, Tel: 0096-1-4427761; Fax: 0096-14427784; Email: mayouf@kfshrc.edu.sa

Submitted: 03 July 2018

Approved: 18 July 2018

Published: 19 July 2018

Copyright: @ 2018 Alqurashi HA, et al. This is an open access article distributed under the Creative Commons Attribution License, which permits unrestricted use, distribution, and reproduction in any medium, provided the original work is properly cited

Keywords: Juvenile idiopathic arthritis; TNFinhibitor; Abatacept; Demyelination neuropathy

Check for updates
Case Report

\section{Biologic therapy-Related demyelinating peripheral neuropathy in a child with Juvenile Idiopathic Arthritis}

\author{
Heba A Alqurashi ${ }^{1}$, Ghada Al-Salmi ${ }^{2}$, Mohammad A Al- \\ Muhaizea ${ }^{1}$ and Sulaiman M Al-Mayouf ${ }^{2 *}$ \\ 'Department of Neuroscience, Pediatric neurology, King Faisal Specialist Hospital and \\ Research Center, Alfaisal University, Riyadh, Saudi Arabia \\ ${ }^{2}$ Department of Pediatric, Pediatric Rheumatology, King Faisal Specialist Hospital and Research \\ Center, Riyadh, Saudi Arabia
}

\section{Abstract}

Demyelinating peripheral neuropathy has been described in association with tumor necrosis factor (TNF) inhibitors. It is rarely developed after treatment discontinuation. We present the case of a child with juvenile idiopathic arthritis who developed peripheral neuropathy few months after TNF inhibitor withdrawal with clinical worsening of the neurological sequelae while undergoing treatment with abatacept.

\section{Introduction}

Children with rheumatic diseases are frequently treated with the traditional and biological disease modifying anti-rheumatic drugs (DMARDs). Fortunately, biological DMARDs opened a new era of management with beneficial therapeutic effect in patients with juvenile idiopathic arthritis (JIA). Among these agents are tumor necrosis factor (TNF) inhibitors, which comprise etanercept and adalimumab [1,2]. Despite, the favorable long-term safety and tolerability profile of TNF inhibitors the accumulating data also showed numerous adverse events. Most of the reported adverse events are mild like infusion related reactions and upper respiratory tract infection, yet serious events such as malignancy and autoimmune diseases have been reported [3-5]. Additionally, rare adverse events including neurological adverse events have been reported after initiation TNF inhibitors treatment. Demyelinating central nervous system is the most frequent serious TNF inhibitors associated neurological adverse events. However, peripheral neuropathies have been reported in the literature $[6,7]$.

We report a child with JIA who developed demyelinating peripheral neuropathy few months after etanercept withdrawal, showing clinical worsening of the neurological sequelae while undergoing treatment with abatacept.

\section{Case Report}

An eight-year-old female diagnosed with polyarticular sub-type JIA at age of six years. The main affected joints were the wrists, knees and ankles. Family history was unremarkable apart from her aunt who is known case of multiple sclerosis. Her initial laboratory results showed normal complete blood count (CBC) with slightly elevated erythrocyte sedimentation rate (ESR) $(25 \mathrm{~mm} / \mathrm{hr})$. Antinuclear antibody and rheumatoid factor were negative. Radiograph of the affected joint did not show arthritic changes. Initially, she was treated with short course of prednisone in addition

How to cite this article: Alqurashi HA, Al-Salmi G, Al-Muhaizea MA, Al-Mayouf SM. Biologic therapyRelated demyelinating peripheral neuropathy in a child with Juvenile Idiopathic Arthritis. Arch Pharm Pharma Sci. 2018; 2: 020-022. https://doi.org/10.29328/journal.apps.1001008 
to methotrexate $(10 \mathrm{mg} /$ week $)$ and etanercept $(0.8 \mathrm{mg} / \mathrm{kg} /$ week). Fortunately, she showed good response and prednisone could be stopped and afterwards etanercept weaned gradually and discontinued. However, few months later she presented with bilateral painful swollen knees and ankles and recurrent falls associated with gait instability and weakness in both legs. Physical examination revealed active synovitis of both knees and ankles. She had a wide base waddling gait with distal upper and lower limbs weakness (3/5 on Medical Research Council-MRC Scale) and generalized areflexia. She had distal intention tremor in upper extremities and dysmetria on finger to nose test but no sensory deficit or cranial nerve involvement.

There was no preceding history of flu-like illness, febrile illness or recent vaccination.

The initial assessment revealed normal CBC, high ESR $(35 / \mathrm{mm})$ with a normal renal and hepatic profile. Because the underlying JIA did not justify the abnormal gait and neurological findings, she underwent nerve conduction study (NCS) and needle electromyography (EMG), which showed conduction slowing, with temporal dispersion and conduction block affecting peroneal and tibial motor nerves but sparing the sural sensory nerve and reduced numbers of motor units in the peroneus longus and tibialis anterior, these findings suggested demyelinating peripheral neuropathy. Unfortunately, a couple of attempts for cerebrospinal fluid taps failed. Magnetic resonance imaging (MRI) of the brain and spine were normal.

Because of the constellation of arthritis and peripheral neuropathy, she received five doses of daily pulse methyl prednisolone therapy $(30 \mathrm{mg} / \mathrm{kg} /$ day), oral weekly methotrexate (12.5 $\mathrm{mg}$ ) and daily intravenous immunoglobulin (IVIG) (0.4gm/ $\mathrm{kg}$ ) for five days. She was in need for biological DMARDs for her arthritis. However, taking in consideration the family history of multiple sclerosis and her demyelinating neuropathy, which could be a latent adverse event from etanercept, she was started on biologic agent with a different mechanism of action. Accordingly, intravenous abatacept infusion initiated for her arthritis as per the usual guidelines $(10 \mathrm{mg} / \mathrm{kg}$ repeated every two weeks then every four weeks). Unfortunately, three weeks later, her neurological symptoms got worse, she had marked hand grip and ankle dorsiflexion weakness $(2 / 5$ on MRC Scale). Repeated NCS and EMG showed worsening findings compared to the first assessment. Abatacept was stopped but she continued four doses of IVIG (1mg/ $\mathrm{kg}$ ) weekly. Fortunately, few weeks later the weakness completely resolved, and her gait improved. Nine months follow-up visit assessment revealed no recurrence of the symptoms with normal neurological examination.

\section{Discussion}

TNF inhibitors related inflammatory neurological disorders are uncommon adverse events. Most of the reported cases of demyelinating central nervous system were optic neuritis and multiple sclerosis. However, demyelinating peripheral neuropathy including Guillain-Barre syndrome and chronic inflammatory polyradiculopathy like disorders have been reported after initiation of TNF inhibitors and considered as induced autoimmune disorders [8,9]. Most of the published data rely on case reports of adult patients and conversely, relatively less frequent in children [6,7]. There is no clear established causal relationship between these medications and the onset of demyelinating manifestations. Additionally, the clinical course of the demyelinating peripheral neuropathy in association with TNF inhibitor use is unclear [6,9]. It is difficult to verify if demyelinating peripheral neuropathy is a direct result of TNF inhibitor treatment or an accidental coincidence particularly in the presence of other potential triggers for instance upper respiratory tract infection or recent vaccination $[8,10]$.

In this report, we presented a child with JIA who developed demyelinating peripheral neuropathy after TNF inhibitor withdrawal and while undergoing treatment with abatacept, which is one of the approved medications for JIA treatment. Abatacept differs from TNF inhibitors as it targets the co-stimulatory signal required for full T-cell activation [11]. 
Demyelinating neuropathy developed early after TNF inhibitor introduction but there is no available data on the onset of peripheral neuropathy after cessation of the treatment. Interestingly, our patient developed peripheral neuropathy few months after discontinuation of TNF inhibitor treatment and experienced worsening of the neurological sequelae while undergoing treatment with abatacept. Although JIA is an autoimmune disease typically not associated with peripheral neuropathy. It is unlikely that the underlying JIA contributed to the development of her neuropathy. To the best of our knowledge, there are no previous reports of demyelinating peripheral neuropathy with abatacept.

Because no other causes of neuropathy could be found and in the absence of other triggers, we postulate that TNF inhibitor was the trigger of the demyelinating process, which further worsened with abatacept, despite the intensive treatment of the neuropathy with prednisone and IVIG.

Overall, patients with demyelinating peripheral neuropathy related to biologic treatment required discontinuation of the potential trigger and providing prednisone and IVIG. Luckily, our patient showed full recovery of the neurological sequelae after abatacept withdrawal and completing the IVIG course.

In summary, demyelinating peripheral neuropathy can represent a rare adverse event in children with rheumatic diseases treated with biologic agents. Patients might present with vague pain and weakness involving the lower extremities, which may be confused with the underlying disease. Therefore, neurophysiological tests are necessary to confirm neuropathy. Increased awareness of the association of demyelinating neuropathy with biologic treatment is required for timely diagnosis and management.

\section{References}

1. Furst D, Keystone E, Braun J, Breedveld F, Burmester G, et al. Updated consensus statement on biological agents for the treatment of rheumatic diseases, 2010. Ann Rheum Dis. 2011; 70: i2-36. Ref.: https://tinyurl.com/ydhu4qoy

2. Davies $\mathrm{R}$, Carrasco R, Foster $\mathrm{H}$, Balidam E, Chieng $\mathrm{S}$, et al. Treatment prescribing patterns in patients with juvenile idiopathic arthritis (JIA): Analysis from the UK Childhood Arthritis Prospective (CAPS). Semin Arthritis Rheum. 2016; 46: 190-195. Ref.: https://tinyurl.com/yayoxrsp

3. Horneff G. Safety of biologic therapies for the treatment of juvenile idiopathic arthritis. Expert Opin Drug Saf. 2015; 14: 1111-1126. Ref.: https://tinyurl.com/y7s7dncp

4. Hashkes $P$, Uziel $Y$, Laxer R. The safety profile of biologic therapies for juvenile idiopathic arthritis. Nat Rev Rheumatol. 2010; 6: 561- 571. Ref.: https://tinyurl.com/y7ov3ckz

5. Al-Mayouf S, Alenazi A, ALJasser H. Biologic agents therapy for Saudi children with rheumatic diseases: indications and safety. Int J Rheum Dis. 2016; 19: 600- 605. Ref.: https://tinyurl.com/ydgjmw76

6. Yagita M, Hamano T, Hatachi S, Fujita M. Peripheral neuropathies during biologic therapies. Mod Rheumatol. 2016; 26: 288-293.

7. Seror R, Richez C, Sordet $\mathrm{C}$, Rist $\mathrm{S}$, Gossec $\mathrm{L}$, et al. Pattern of demyelination occurring during anti-TNF- therapy: a French national survey. Rheumatology (Oxford). 2013; 52: 868-874. Ref.: https://tinyurl.com/yc8ayp7k

8. Ramos-Casals M, Roberto-Perez-Alvarez, Diaz-Lagares C, Cuadrado M, Khamashta M. Autoimmune diseases induced by biological agents: a double-edged sword? Autoimmun Rev. 2010; 9: 188-193. Ref.: https://tinyurl.com/y95jxhna

9. Perez-Alvarez R, Pérez-De-Lis $M$, Ramos-Casals M. Biologics-induced autoimmune diseases. Curr Opin Rheumatol. 2013; 25: 56-64. Ref.: https://tinyurl.com/yd7hpn5p

10. Alvarez-Lario B, Prieto-Tejedo R, Colazo-Burlato $M$, Macarron-Vicente J. Severe Guillain-Barre syndrome in a patient receiving anti-TNF therapy. Consequence or coincidence. A case-based review. Clin Rheumatol. 2013; 32: 1407-1412. Ref.: https://tinyurl.com/ybf9zbmt

11. Herrero-Beaumont G, Martínez Calatrava M, Castañeda S. Abatacept mechanism of action concordance with its clinical profile. Reumatol Clín. 2012; 8: 78-83. 